\title{
Topology Mapping using Multidimensional Scaling to Improve Routing Quality
}

\author{
Poornima G. \\ Dept of Electronics and \\ Communication \\ Engineering \\ BMS College of \\ Engineering, Bangalore, \\ India
}

\author{
Suresh Babu \\ Department of Computer \\ Science and Engg \\ UVCE, Bangalore
}

\author{
K. B. Raja \\ Department of Computer \\ Science and Engg \\ UVCE, Bangalore
}

\author{
K. R. Venugopal \\ Department of Computer \\ Science and Engg \\ UVCE, Bangalore
}

\begin{abstract}
The proposed Enhanced Topology Aware Routing (ETAR) procedure efficiently maps a network topology into a lowdimensional virtual coordinate space. The hop distances between node pairs are preserved. The node is categorized by a coordinate vector. This vector contains hop distances to the anchor nodes. Therefore ETAR can assist greedy forwarding to find the precise neighbor one hop closer to the terminus by eliminating the local minimum problem and attain high success ratio of packet delivery and throughput without location information. Creating VCs involve a single flooding for each anchor and each collecting coordinates from a set of small number of random nodes hence reduces the average end to end delay, energy and power. Further, embedding a network topology based on the metric of Probable Communication Count (PCC) improves the routing quality.
\end{abstract}

\section{Keywords}

Wireless Sensor and Actor Networks, Virtual Coordinate System, Topology Mapping, Multidimensional Scaling, Routing.

\section{INTRODUCTION}

In recent years application specific Wireless Sensor Networks require point to point routing provision rather than a tree based many to one routing approach. Routing protocols for Wireless Sensor Networks (WSNs) can be broadly classified into physical coordinate-based and virtual coordinate-based schemes. Physical domain routing relies on the physical (geographic) position information for routing, e.g., as in geometrical routing [1]. Virtual domain (or logical) routing is based on a set of virtual coordinates that capture the position and route information, e.g., hierarchical/clustering schemes and Virtual Coordinate (VC) based routing[2].

The Virtual Coordinate System (VCS) addressing scheme does not require GPS or additional hardware for signal strength measurements. The VC routing is less susceptible to local minima as the connectivity information is embedded in each ordinate. The number of anchors becomes the networks' dimensionality. Anchor placement and the performance of VCR are highly correlated.

Finding the overall best anchor placement is extremely challenging and, even if found, it is not likely to eliminate the local minima problem except in a limited set of network topology classes as 1D networks and 2D full grids.

\section{2. BACKGROUND}

Topology Aware Routing for local minimum problem is suggested in [3]. TAR guides the GF along the near-optimal paths in terms of global metrics. The topology awareness is achieved via constructing a Virtual Coordinate Space (VCS) where the geometric distance between two arbitrary nodes reflects the actual distance in corresponding global metric space. Bao et al., [4] proposed a highly scalable cluster-based hierarchical trust management protocol for WSNs to effectively deal with selfish or malicious nodes. The scheme uses multidimensional trust attributes to evaluate the overall trust of a sensor node for trust-based geographic routing and trust-based intrusion detection. Jun-Won $\mathrm{Ho}$ et al., [5] proposed a zone-based node compromise detection and revocation scheme in WSNs. The scheme focus on the use sequential hypothesis testing to detect suspect regions in which compromised nodes are likely placed. Additionally, they modeled the detection problem using a game theoretic analysis. Sirajul Ameen et al., [6] proposed to reduce the burden of cluster head and place relay node in between head and sink. Poisson distribution is used to predict the energy level of the each node in the cluster. Haidong Yuan et al., [7], have discussed a new three-level coordination model on a hierarchical geographical clustering paradigm. The cluster is designed by isolating the action area into fixed zones to form a virtual grid, so that the functioning area and workload can be optimally split up into different actors. Senel et al., [8], proposed a distributed algorithm for establishing a connected inter-actor network topology. The actor network is initially partitioned with intra-connected sub-networks, the algorithm pursues a coordinated actor movement to both minimize the total and maximum travel distances of the individual actors by considering the least connected dominating set of each subnetwork. Chen Qian and Simon S. Lam [9] proposed greedy distance vector routing, a geographic routing protocol to improve end-to-end path costs using latency, expected transmission count, and expected transmission time which capture network and link characteristics as additive routing metric.

Mohamed Lehsaini et al., [10], discussed solutions for the issues of reduced communication and processing overhead. Efficient Cluster-Based Fault-Tolerant Schemes, ECFS-1 tolerates link failures and ECFS-2 tolerates both sensor faults and link failures. A clustered architecture with primary cluster-head and secondary one are used to avoid node failure. Md Zakirul et al., [11] addressed the computing system issues and civil engineering techniques in conjunction. The Fault Tolerance for Structural Health Monitoring searches the repairing points in clusters and places a set of backup sensors at those points by satisfying civil engineering requirements. The algorithm exploiting sensor decentralized computing i.e., is potentially suitable for communication constrained. 


\section{PROPOSED METHODLOGY 3.1 Topology Information}

The concept of Multi-Dimensional Scaling (MDS) is used to reduce the dimension of a network topology. The newly formed virtual topology reduces network space thereby reducing the routing length. The base station gathers the information of the existing topology by transmitting a request packet to all the nodes in the network. The actor nodes assist in the information gather and report it, back to the base station. Distributed network with $\mathrm{N}$ nodes randomly placed in a network area of $500 \mathrm{~m} * 500 \mathrm{~m}$, out of which $\mathrm{M}$ nodes are randomly chosen as anchors.

The anchors propagates beacon message to trace the hop count value to all node in the network. Based on the trace value received the node gets an estimate of its hop distance to the anchor. The node will communicate to the anchor that has least hop distance to it.

The anchors will report the constructed hop count matrix using Equation 1 to the base station.

$\mathrm{H}=\left[\mathrm{h}_{1} \mathrm{~h}_{2} \mathrm{~h}_{3} \ldots \mathrm{h}_{\mathrm{M}}\right]$

The matrix is now mapped to a low dimensional two norm Euclidean space by the base station and the new coordinates are assigned to the anchor forwarded to the nodes. Now the nodes are repositioned virtually. The node that are not localized by anchors calculates its virtual coordinate by itself using the least square fitting method to ensure that the differences between hop distances and the corresponding Euclidean distances from the node to all anchors are minimized

\subsection{Shortest Path Calculation}

Once the base station receives the report from the anchor it computes the minimum distances in terms of hops between any node pair. The minimum distance calculation is done using Dijkstra's algorithm.

\subsection{Gradient Algorithm for Repositioning}

The Gradient starts by anchor nodes and helps un-localized nodes to estimate their positions from three anchor nodes using tri-lateration. The hop count value is used which is initially set to 0 and then incremented, when it propagates to other neighboring nodes. Every sensor node takes information of the shortest path from anchor nodes.

The Gradient algorithm calculates the new node positions as follows:

(i) The anchor node $(a, b, c)$ broadcasts beacon message containing its coordinate and hop count value.

(ii) After this the un-localized node computes the shortest path between itself and the anchor node from which it receives beacon signals.

(iii) Using the trilateral positioning, the new distance $\left(\mathrm{d}_{\mathrm{a}}, \mathrm{d}_{\mathrm{b}}\right.$, $d_{c}$ ) of the un-localized node with respect to the three anchor nodes that are not collinear is calculated by using following formulae 2 to 4 ,

$d_{a}=\sqrt{\left(x-x_{a}\right)^{2}+\left(y-y_{a}\right)^{2}}$

$d_{b}=\sqrt{\left(x-x_{b}\right)^{2}+\left(y-y_{b}\right)^{2}}$

$d_{c}=\sqrt{\left(x-x_{c}\right)^{2}+\left(y-y_{c}\right)^{2}}$
Using equation (5) the new position of the un-localized node is estimated.

$$
\begin{aligned}
{\left[\begin{array}{l}
X \\
Y
\end{array}\right]=} & \frac{1}{2}\left[\begin{array}{ll}
\left(x_{a}-x_{c}\right) & \left(x_{b}-x_{c}\right) \\
\left(y_{a}-y_{c}\right) & \left(y_{b}-y_{c}\right)
\end{array}\right] * \\
& {\left[\begin{array}{l}
x_{a}^{2}+y_{a}^{2}-x_{c}^{2}-y_{c}^{2}-d_{a}^{2}-d_{c}^{2} \\
x_{b}^{2}+y_{b}^{2}-x_{c}^{2}-y_{c}^{2}-d_{b}^{2}-d_{c}^{2}
\end{array}\right] }
\end{aligned}
$$

The new distances of the nodes with respect to the anchors are computed using the equation (6)

$$
d_{i j}^{1}=h_{i j} * d_{h o p}
$$

New Distance $\left(n_{i j}\right)=\sum_{x_{i} x_{j \in x}}\left(d_{i j}-d_{i j}^{1}\right) \ldots$

The new distances for the nodes with respect to the anchors is computed using original distance and new distance using Equations 7. This distance is propagated to anchor from base station thus the reduced path is used for the transmission of data packets.

\section{PROBLEM DEFINITION}

To encodes a network topology into a low-dimensional virtual coordinate space where hop distances between pairwise nodes are preserved.

The objectives are:

(i) To construct virtual coordinate based on minimum hop counts.

(ii) Mapping a distributed network topology using MDS.

(iii) To provide an improved routing performance.

Table 4.1: Enhanced Topology Aware Routing (ETAR)

\begin{tabular}{|l|l|}
\hline Input & Anchors to circulate connectivity request packet \\
\hline Output & Base Station to circulate new virtual coordinates \\
\hline Step 1 & To obtain the topology information \\
\hline Step 2 & $\begin{array}{l}\text { Calculate the shortest path distances between the } \\
\text { nodes and the anchors }\end{array}$ \\
\hline Step 3 & Reposition the nodes use the gradient algorithm \\
\hline Step 4 & $\begin{array}{l}\text { Calculate the new distance with respect to the } \\
\text { anchor nodes }\end{array}$ \\
\hline Step 5 & $\begin{array}{l}\text { Choose the new distance such that the difference } \\
\text { between }\end{array}$ \\
\hline
\end{tabular}

\section{RESULTS AND DISCUSSION}

To study the performance of the proposed method the network of area $500 \mathrm{~m} * 500 \mathrm{~m}$ is simulated using NS-2 with 100 nodes deployed. The node parameter setting is as per Mica $\mathrm{Z}$ mote that is equipped with an Atmel ATmega128L and has a CC1000 transceiver. Wireless channel and Omni-directional antenna for transmission and reception of signal is defined. MAC type is 802.11 with Droptail/Priqueue, with two ray ground propagation type. The traffic generator is UDP; it accepts data in variable size chunks and uses RTP timestamp. The proposed scheme is a time based mechanism and on demand routing where the data transmission takes place at high rate for entire duration. The performance parameters are Packet delivery ratio (PDR), throughput, end to end delay and average path length. The original network topology is compressed virtually. Multidimensional scaling forms virtual 
positioning for the nodes with minimum distance. i.e., it maps global hop distance space into two norm Euclidean space.

Table 4.2 shows the old and new (X, Y) coordinates for a hundred node network deployment scenario. It is also observed that for any node $\mathrm{n}_{\mathrm{i}}$ the $(\mathrm{X}, \mathrm{Y})$ node co-ordinates are relocated and over all the positioning of the nodes is reduced due to the mapping of the original topology into Euclidean space based on the metric of probable communication count (PCC).

Table 4.2: Repositioning of nodes with MDS

\begin{tabular}{|c|c|c|c|c|}
\hline $\begin{array}{c}\text { Node ID } \\
\mathrm{n}_{\mathrm{i}}\end{array}$ & \multicolumn{2}{|c|}{$\begin{array}{c}\text { Node Position } \\
\text { without MDS }\end{array}$} & \multicolumn{2}{c|}{$\begin{array}{c}\text { Node Position } \\
\text { with MDS }\end{array}$} \\
\cline { 2 - 5 } & $\begin{array}{c}\mathrm{x} \\
\mathrm{X}- \\
\text { ordinate }\end{array}$ & $\begin{array}{c}\mathrm{y} \\
\text { Co- } \\
\text { ordinate }\end{array}$ & $\begin{array}{c}\mathrm{x} \\
\mathrm{x}- \\
\text { ordinate }\end{array}$ & $\begin{array}{c}\mathrm{y} \\
\text { Co- } \\
\text { ordinate }\end{array}$ \\
\hline 0 & 39.432 & 395.502 & 125 & 125 \\
\hline 2 & 6.6536 & 166.776 & 20.6257 & 148.841 \\
\hline 13 & 433.02 & 125.882 & 104.695 & 163.25 \\
\hline 30 & 220.898 & 206.664 & 160.274 & 124.657 \\
\hline 41 & 45.626 & 79.452 & 156.99 & 104.671 \\
\hline 56 & 193.414 & 432.916 & 172.875 & 182.63 \\
\hline 62 & 108.247 & 5.240 & 130.346 & 159.73 \\
\hline 74 & 338.180 & 87.394 & 123.65 & 135.581 \\
\hline 83 & 263.748 & 328.463 & 78.32 & 86.1173 \\
\hline 93 & 414.016 & 423.509 & 71.48 & 71.3172 \\
\hline & & & & \\
\hline
\end{tabular}

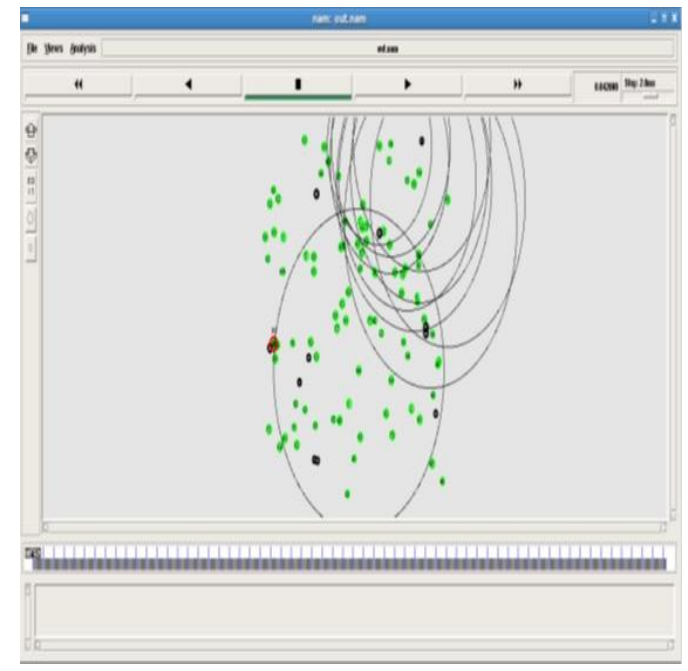

Figure 4.1: NAM trace for the original topology (without MDS)

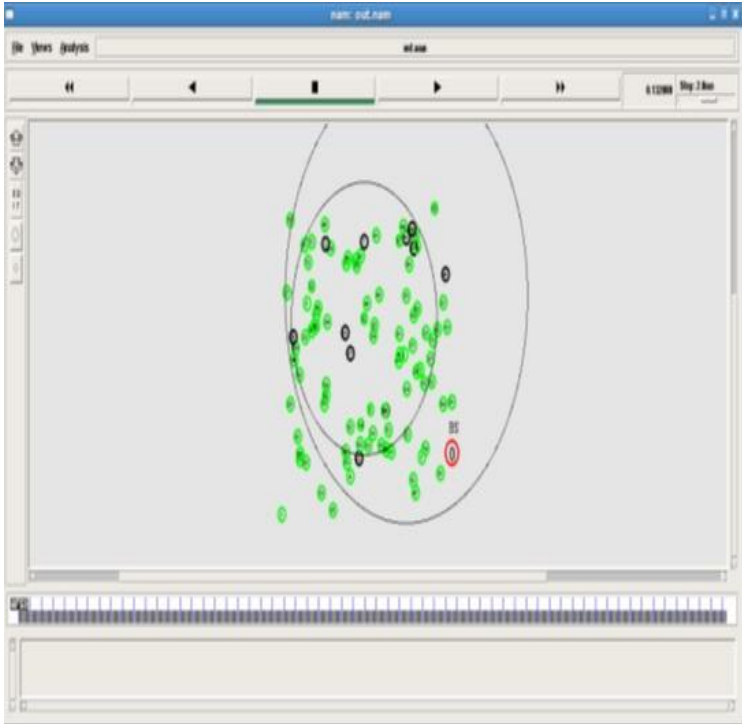

Figure 4.2: NAM trace for the virtual topology (with MDS)

Figure 4.1 and 4.2 shows the NAM trace for the network topology without and with multidimensional scaling. It is observed that the Virtual Coordinate Systems (VCS) characterize each node by a coordinate vector consisting of the shortest path hop distances to a subset of nodes, named anchors.

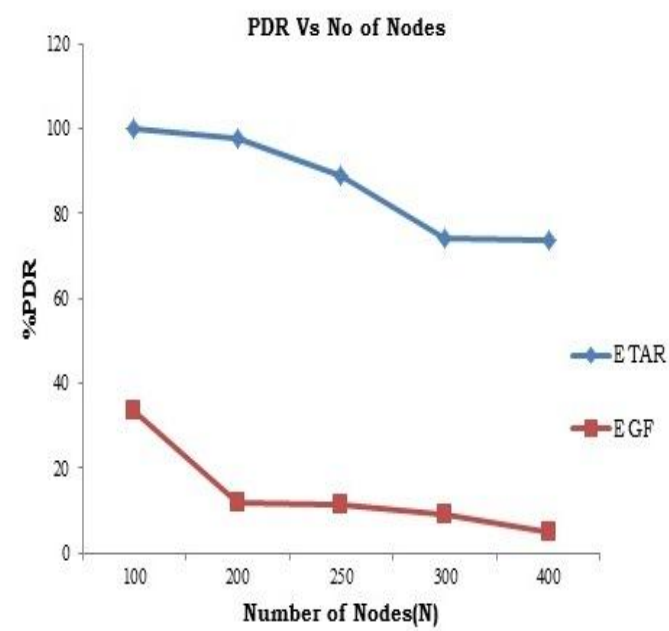

Figure 4.3: Comparison of PDR for EGF and ETAR 


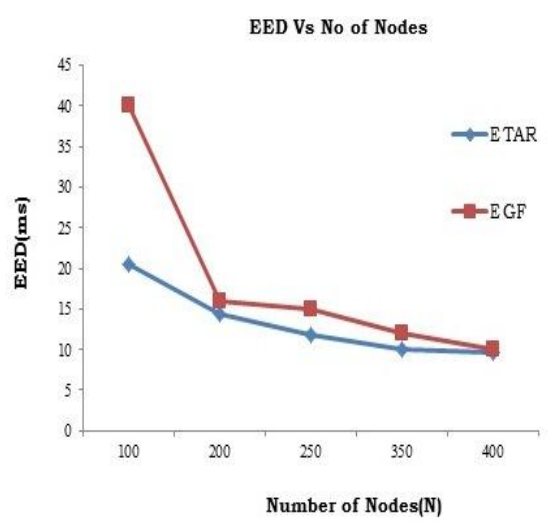

Figure 4.4: Comparison of EED for EGF and ETAR

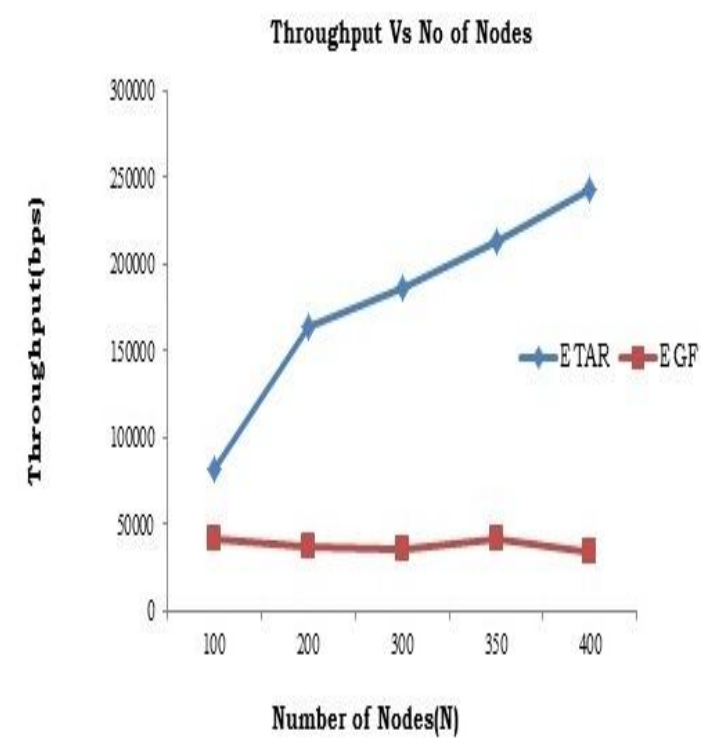

Figure 4.5: Comparison of Throughput for EGF and ETAR

Figure 4.3 to 4.5 shows that the proposed ETAR has better performance in terms of PDR, throughput, average end to end delay, and average routing length compared to EGF. It is seen that the proposed ETAR increases the packet delivery ratio, the packets are transmitted through the anchor nodes which are placed based on the virtual coordinates, therefore the PDR is high as the path failure is less hence it is suitable for real time application. The packet delivery ratio on an average increases by $72.56 \%$ with an end to end delay reduced by $5.42 \%$ as compared to the existing work.
APL Vs No of Nodes

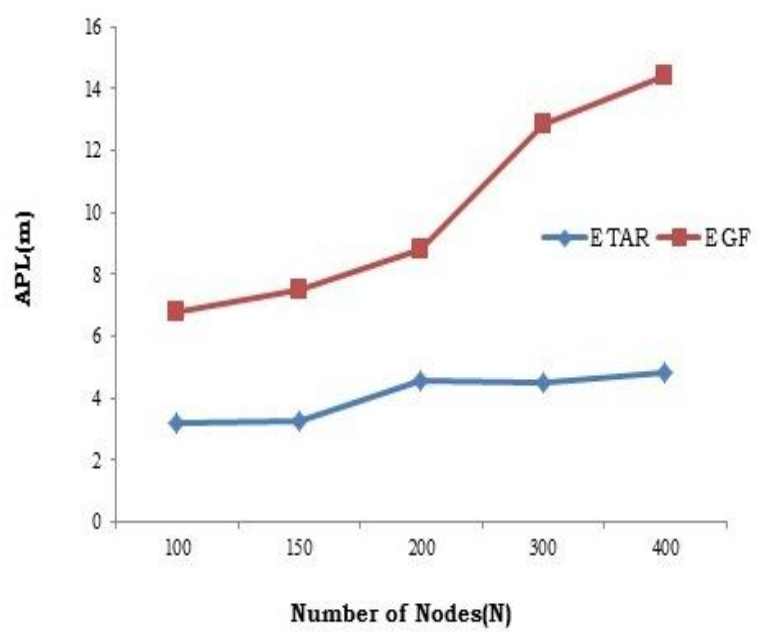

Figure 4.6: Comparison of Average Path Length for EGF and ETAR

The average routing length in figure 4.6 is very less when compared to the existing technique, because the virtual coordinates obtained are placed in reduced area

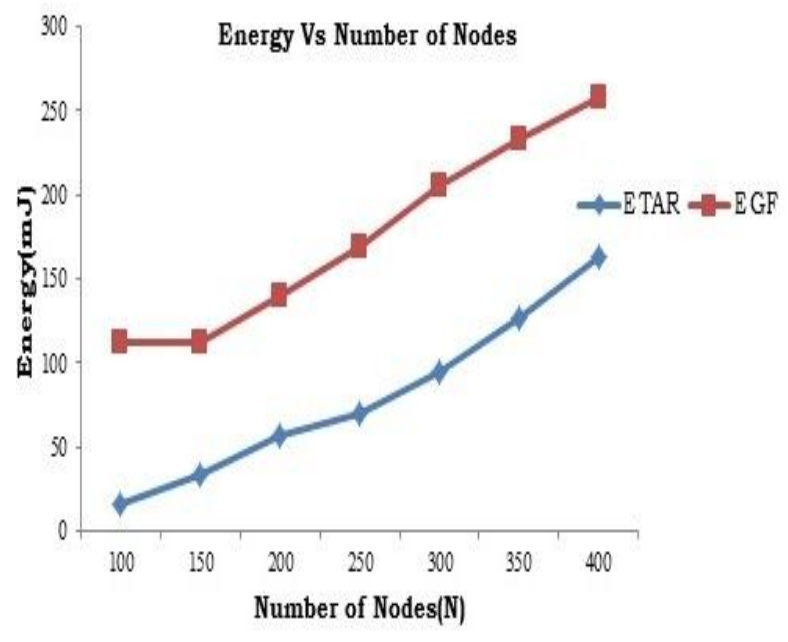

Figure 4.7: Comparison of Energy for EGF and ETAR.

The energy consumption in generating the topological coordinates is significantly lower than that in generating physical coordinates as shown in figure 4.7. Creating VCs involve a single flooding for each anchor, and each collecting coordinates from a set of small number of random nodes. As energy and power are directly proportional to each other, the power of the proposed algorithm is also less than the existing algorithm as seen in figure 4.8 . 


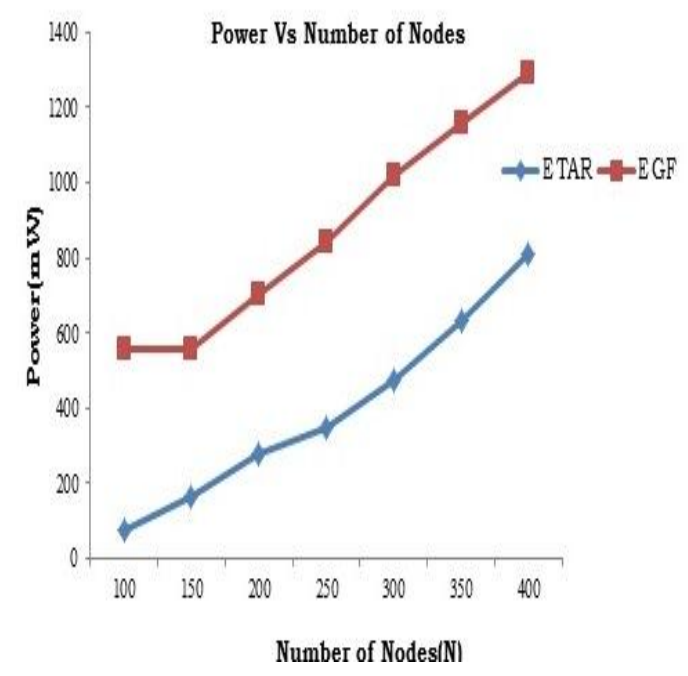

Figure 4.8: Comparison of Power for EGF and ETAR

Table 4.3 shows the performance in terms of PDR, throughput, average end to end delay, average routing length, energy and power for a network with 100 nodes. The number of anchors is varied in order to analyze the dimensionality of the network. It is observed that the success ratio of packet delivery improves with increase in number of anchors and also the average routing length reduces showing the effectiveness of the enhanced TAR in improving routing quality. Hence the proposed protocol is best suited for burst traffic applications like real time application, multimedia, video conferencing etc.

Table 4.3: Performance by varying the No of Anchors $(\mathrm{N}=100)$

\begin{tabular}{|c|c|c|c|c|c|c|}
\hline $\mathrm{M}$ & $\begin{array}{c}\text { PDR } \\
(\%)\end{array}$ & $\begin{array}{c}\mathrm{S} \\
(\mathrm{bps})\end{array}$ & $\begin{array}{c}\text { EED } \\
(\mathrm{ms})\end{array}$ & $\begin{array}{c}\mathrm{E} \\
(\mathrm{J})\end{array}$ & $\begin{array}{c}\text { P } \\
(\mathrm{mW})\end{array}$ & $\begin{array}{c}\text { ARL } \\
(\mathrm{m})\end{array}$ \\
\hline 2 & 87.43 & 71669 & 22.66 & 19.68 & 98 & 4.33 \\
\hline 3 & 87.43 & 71669 & 22.66 & 19.68 & 98 & 4.33 \\
\hline 4 & 88.31 & 72395 & 22.44 & 19.28 & 96 & 4.10 \\
\hline 5 & 88.6 & 72629 & 22.36 & 18.85 & 94 & 4.04 \\
\hline 6 & 89.51 & 73379 & 22.13 & 18.07 & 90 & 4.01 \\
\hline 7 & 90.8 & 74456 & 21.81 & 17.07 & 85 & 3.96 \\
\hline 8 & 91.4 & 74925 & 21.68 & 16.87 & 84 & 3.95 \\
\hline 9 & 95.48 & 78274 & 20.75 & 16.06 & 80 & 3.90 \\
\hline 10 & 99.81 & 81820 & 20.05 & 15.64 & 78 & 3.22 \\
\hline
\end{tabular}

A dense and uniform anchor distribution is helpful to minimize the distance estimation error. However, increasing anchor density will lead to higher dimensionality of the embedded space. In order to achieve high embedding accuracy while preserving low dimensionality of the embedded space, ETAR uses sufficient number of anchors to abstract the network topology.

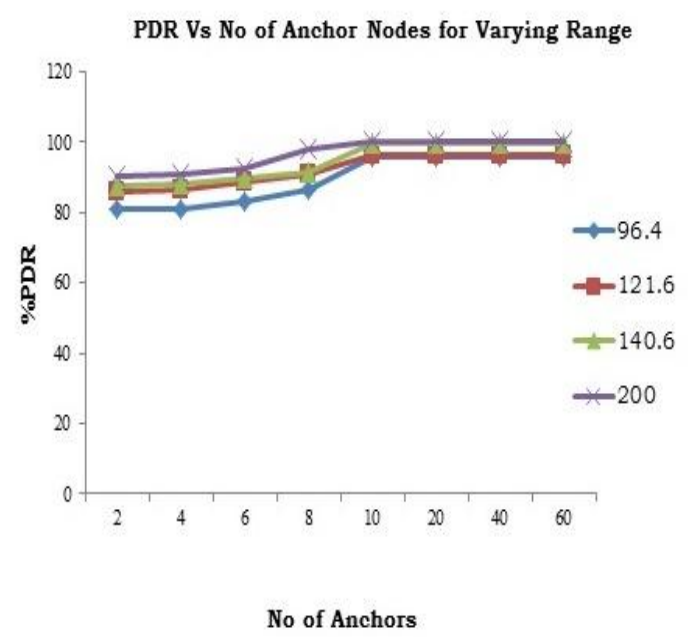

Figure 4.9: Comparison of \%PDR with number of Nodes for EGF and ETAR

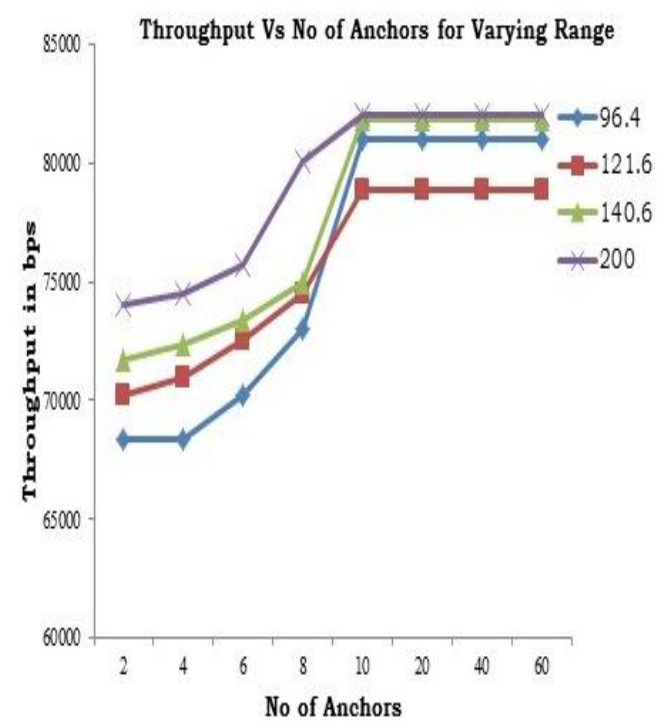

Figure 4.10: Comparison of Throughput with number of Nodes for EGF and ETAR

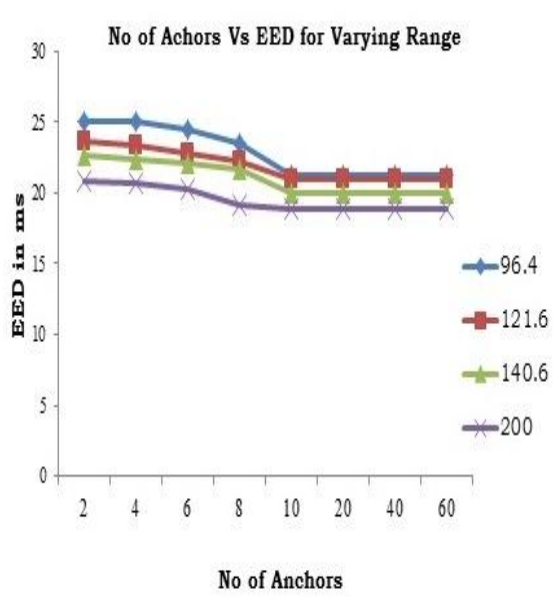

Figure 4.11: Comparison of EED with number of Nodes for EGF and ETAR 


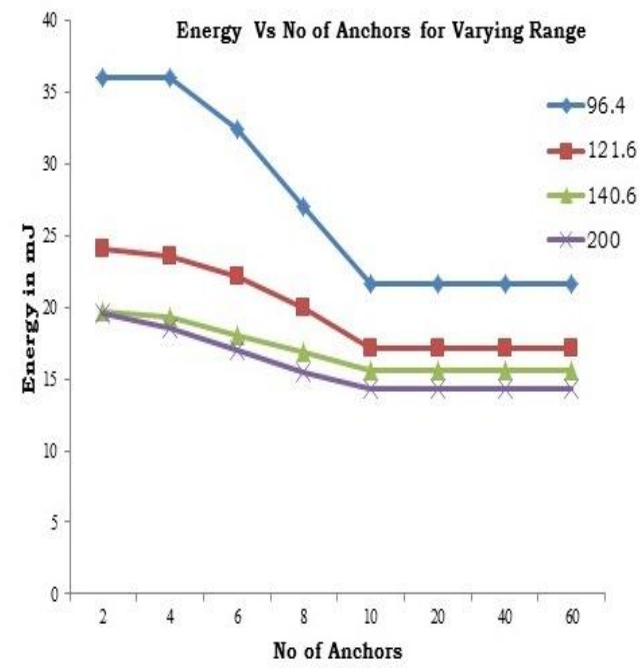

Figure 4.12: Comparison of ARL with number of Nodes for EGF and ETAR

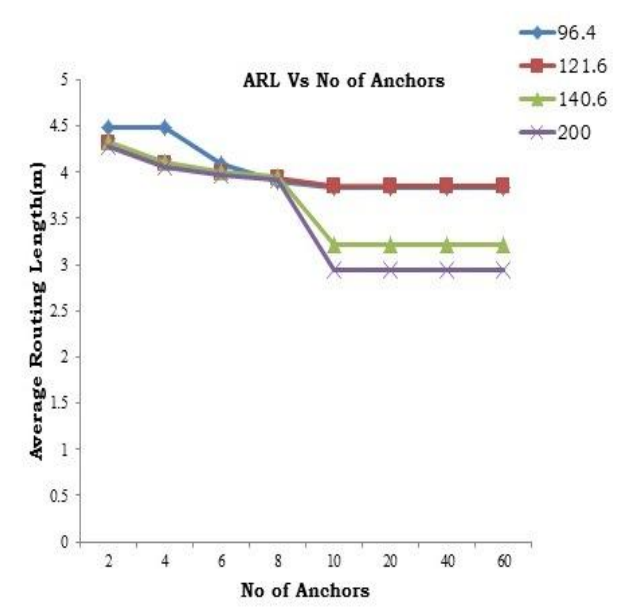

Figure 4.13: Comparison of ENERGY with number of Nodes for EGF and ETAR

Power Vs No of Anchors for varying Range

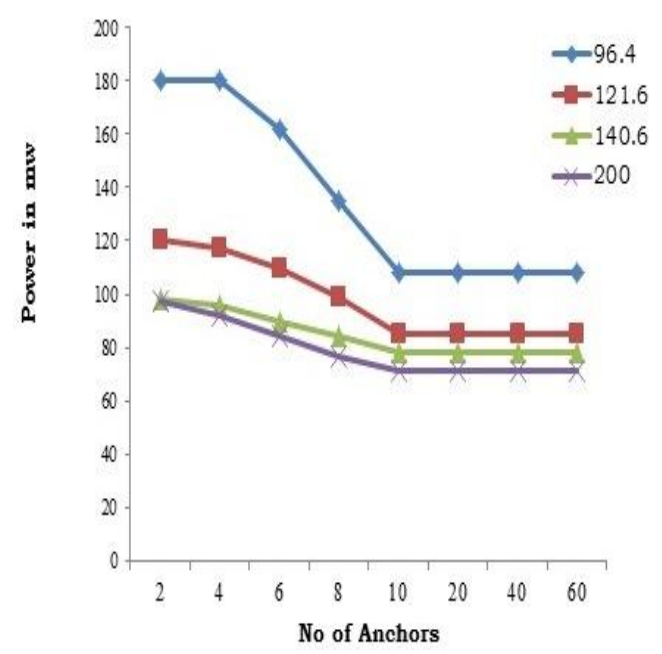

Figure 4.14: Comparison of POWER with number of Nodes for EGF and ETAR
Figure 4.9 to 4.14 shows the performance of the ETAR for varying range and it seen that it performs better for $r=200 \mathrm{~m}$. Physical design statistics such as physical voids and even relative physical positions of sensor nodes with respect to $\mathrm{X}$ $\mathrm{Y}$ directions independent of physical information or signal strength measurements are obtained. The capacity to restore directionality and topology-based Cartesian coordinates makes the scheme a better alternative to geographic coordinates. The performance of the proposed algorithm is improved as it adapts a scalable approach that avoids the need for a table driven method and recovery scheme. The virtual coordinate for each node is based on minimum count from the anchor node. The probable communication count is proportional to the geometric distance in space.

\section{CONCLUSION}

Routing protocol technique which provides fast delivery of data, reduced energy and power. It uses virtual coordinates without the need for expensive and unreliable localization techniques. The length of the coordinate, local minima problem and identical coordinate problem are directly correlated with the anchor placement. Properties and issues of VCS's are investigated. This protocol is best suitable for providing QoS, the QoS parameters that is end to end delay, throughput and packet delivery ratio for maximum bust size. It supports delay sensitive traffic, efficiently manages the limited resources that is available to the network. The TAR algorithm is applied for randomly deployed static nodes using the centralized method. It can be applied for grid network with mobile nodes using the decentralized method to further improve the network performance parameters. The characteristics of small average path length in WSN are beneficial to reduce communication costs, save node energy and make the network exhibit a good robust under random attack.

\section{REFERENCES}

[1] J N Al-Karaki, and A E Kamal, "Routing Techniques in Wireless Sensor Networks: A survey", IEEE Transaction on Wireless Communications, Vol. 11, pp. 6-28, 2004

[2] A Caruso, S Chessa, S De, and A Urpi, "GPS free Coordinate Assignment and Routing in Wireless Sensor Networks", IEEE Joint Conference of Computer and Communications Societies, Vol. 1,pp. 150- 160, 2005.

[3] Pei Huang, Chen Wang, and Li Xiao, "Improving Endto-End Routing Performance of Greedy Forwarding in Sensor Networks", IEEE Transactions on Parallel and Distributed Systems, vol. 23,no. 3, pp. 556-563, 2012.

[4] F Bao, I R Chen, M Chang, and J H Cho, "Hierarchical Trust Management for Wireless Sensor Networks and Its Applications to Trust-based Routing and Intrusion Detection", IEEE Transactions on Network and Service Management, vol. 9, no. 2,pp.169-183, 2012.

[5] J Ho, M Wright, and S K Das, "Zone Trust: Fast Zonebased node compromise Detection and Revocation in Wireless Sensor Networks using Sequential Hypothesis Testing", IEEE Transactions on Dependable And Secure Computing, vol. 9, no. 4, pp.494-510, 2012.

[6] Sirajul Ameen, Mohammed Ashraf and Prabakaran, "Fault Tolerance using Cluster in Wireless Sensor network", International Journal of Advanced Research in Computer Science and Software Engineering, vol. 4, no. 4, pp. 351-356, 2014. 
[7] Haidong Yuan, Huadong $\mathrm{Ma}$ and Hongyu Liao, "Coordination Mechanism in Wireless Sensor and Actor Networks", ACM International Multi-Symposiums on Computer and Computational Sciences, pp. 627-634, 2006.

[8] Senel, Akkaya and M Younis, "An Efficient Mechanism for Establishing Connectivity in Wireless Sensor and Actor Networks", IEEE International Conference on Global Telecommunications, pp. 1129-1133, 2007.

[9] Chen Qian and Simon S Lam, "Greedy Distance Vector Routing", International Conference on Distributed Computing Systems, pp.857-868, 2011.

[10] Mohamed Lehsaini, Mohammed Feham and Herve Guyennet,"Efficient Cluster-based Fault-tolerant Schemes for Wireless Sensor Networks", IEEE International Conference on New Technologies, Mobility and Security, pp. 1-5,2012.

[11] Md Zakirul, Alam Bhuiyan, Jiannong Cao and Guojun Wang,"Deploying Wireless Sensor Networks with Fault Tolerance for Structural Health Monitoring", IEEE International Conference on Distributed Computing in Sensor Systems, pp. 194-202, 2012.

\section{AUTHOR'S PROFILE}

Poornima.G received the $\mathrm{BE}$ degree in Electronics and Communication Engineering from Bangalore University and the M.E. degree in Digital Communication from Bangalore University, Bangalore. She was awarded Ph.D. in Computer Science and Engineering at University Visvesvaraya College of Engineering, Bangalore University. She is currently an Associate Professor, Dept. of Electronics and Communication Engineering, BMS College of Engineering. Bangalore. Her research interests include Computer Networks and Signal Processing. She has twenty research publications in refereed International Journal and Conference Proceedings. She is a life member of Indian Society for Technical Education, New Delhi.

Suresh Babu is Professor, Dept. of Electronics and Communication Engineering, University Visvesvaraya College of Engineering, Bangalore University, Bangalore. He obtained his BE and ME in Electronics and Communication
Engineering from University Visvesvaraya College of Engineering, Bangalore. He was awarded Ph.D. in Computer Science and Engineering from Bangalore University. He has over thirty research publications in refereed International Journals and Conference Proceedings. His research interests include Image Processing, Biometrics, Signal Processing, and Computer Networks.

K B Raja is Professor, Dept. of Electronics and Communication Engineering, University Visvesvaraya College of Engineering, Bangalore University, Bangalore. He obtained his BE and ME in Electronics and Communication Engineering from University Visvesvaraya College of Engineering, Bangalore. He was awarded Ph.D. in Computer Science and Engineering from Bangalore University. He has over eighty research publications in refereed International Journals and Conference Proceedings. His research interests include Image Processing, Biometrics, VLSI Signal Processing, and Computer Networks.

$\mathrm{K} \mathrm{R}$ Venugopal is currently the Principal, University Visvesvaraya College of Engineering, Bangalore University, Bangalore. He obtained his Bachelor of Engineering from University Visvesvaraya College of Engineering. He received his Master's degree in Computer Science and Automation from Indian Institute of Science, Bangalore. He was awarded $\mathrm{Ph} . \mathrm{D}$. in Economics from Bangalore University and Ph.D. in Computer Science from Indian Institute of Technology, Madras. He has a distinguished academic career and has degrees in Electronics, Economics, Law, Business Finance, Public Relations, Communications, Industrial Relations, Computer Science and Journalism. He has authored 27 books on Computer Science and Economics, which include Petrodollar and the World Economy, C Aptitude, Mastering $\mathrm{C}$, Microprocessor Programming, Mastering C++ etc. He has been serving as the Professor and Chairman, Department of Computer Science and Engineering, University Visvesvaraya College of Engineering, Bangalore University, Bangalore. During his three decades of service at UVCE he has over 250 research papers to his credit. His research interests include computer networks, parallel and distributed systems, digital signal processing and data mining. 